تأثير إضافة النيتروجين، الفوسفور و البوتاسيوم والسماد العضوي على بعض صفات ثمار

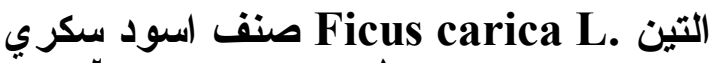

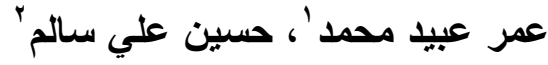

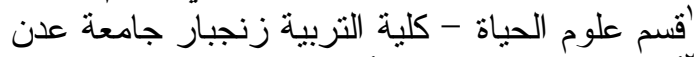

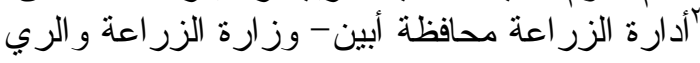

Received on: 28/9/2016

Accepted for publication on: 4/10/2016

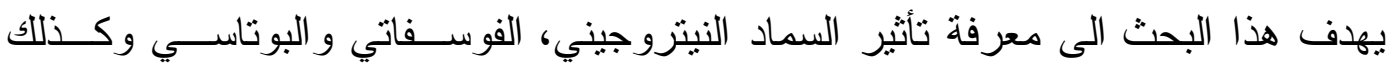

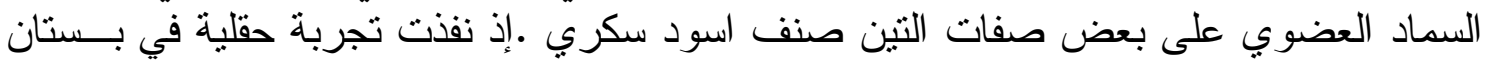

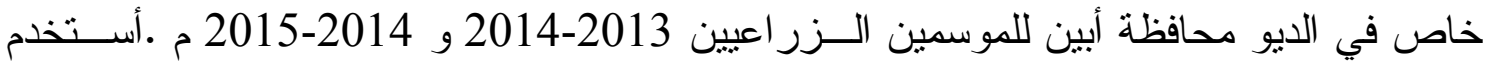

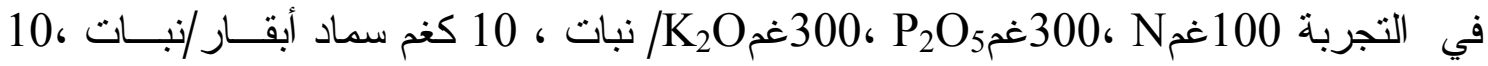

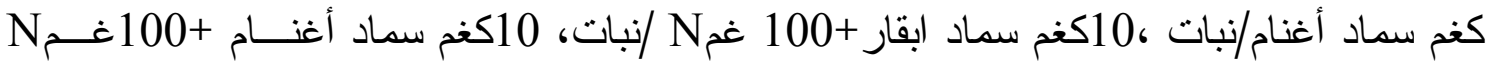

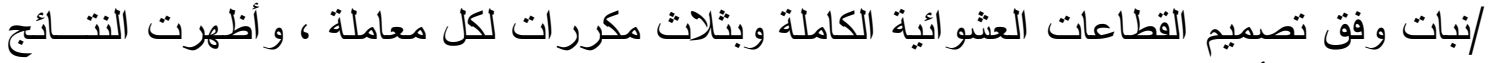

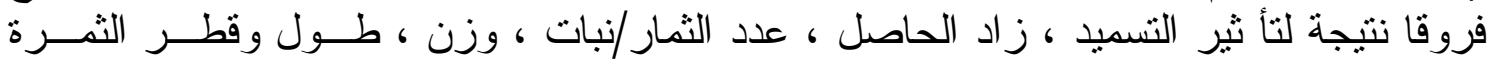

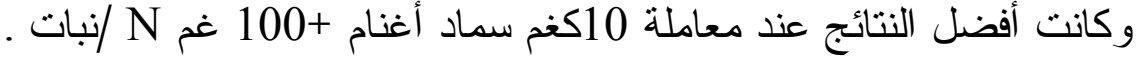

كلمات مفتاحيه: التين ، تغذية معدنية ، تسميل عضوي .

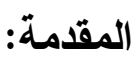

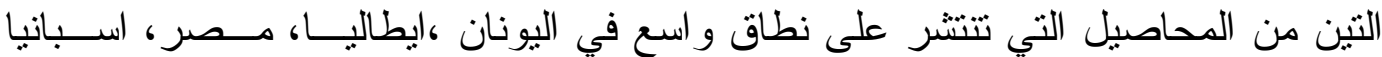

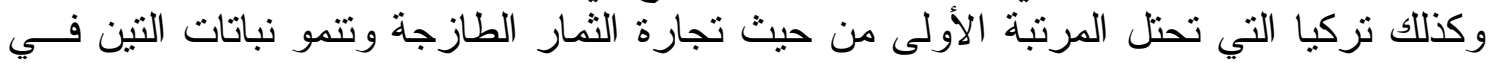

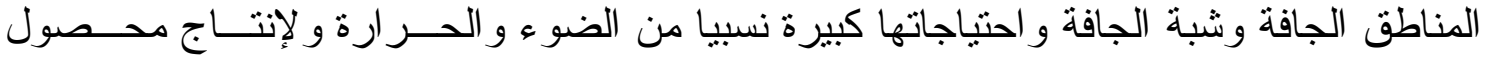

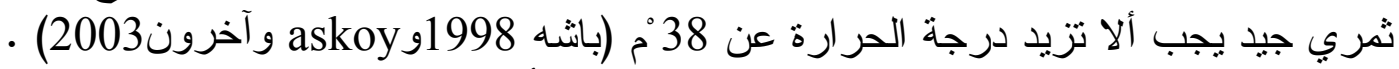

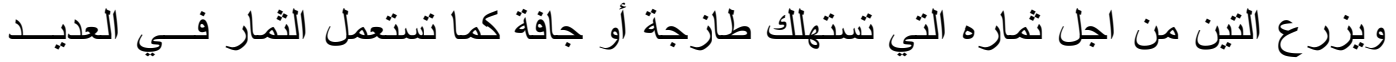

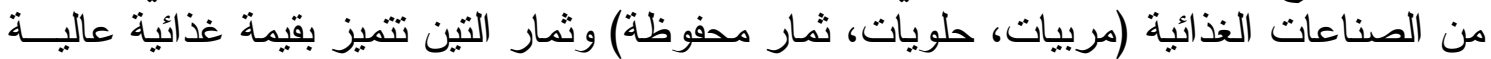

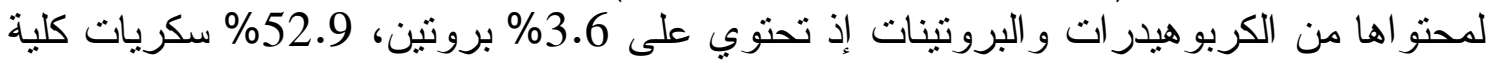

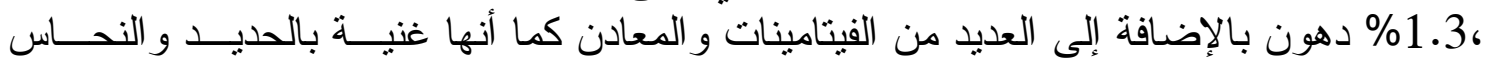

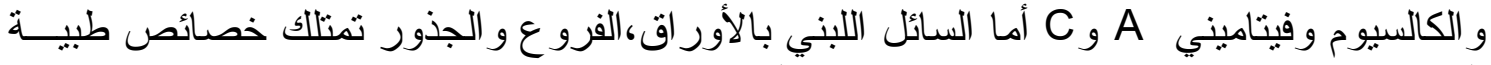

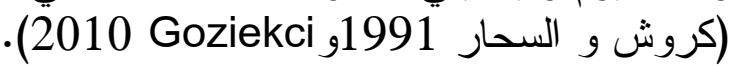

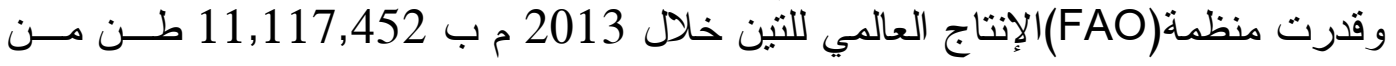

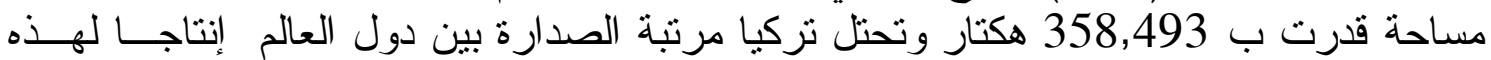

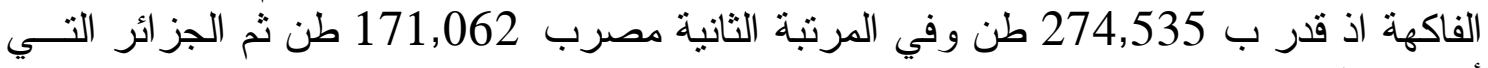
أنتجت

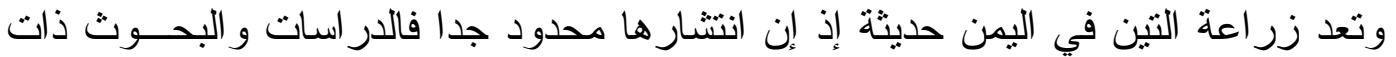

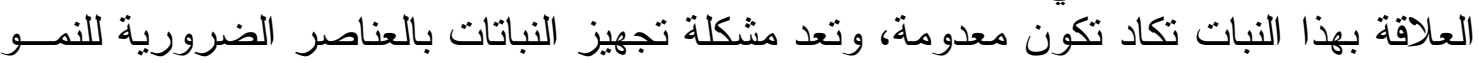

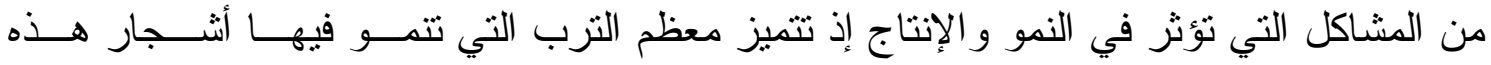

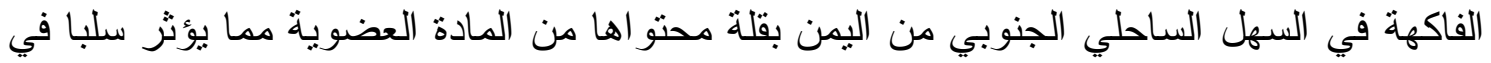

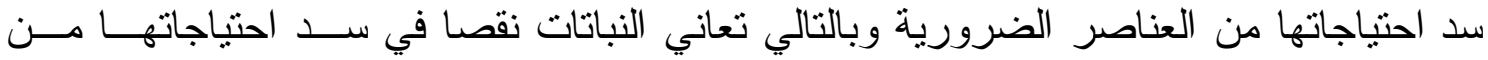

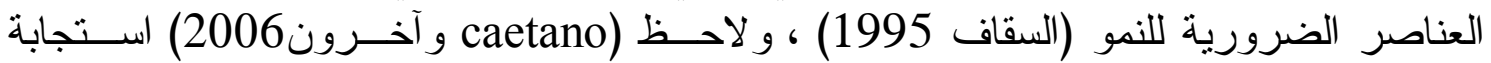

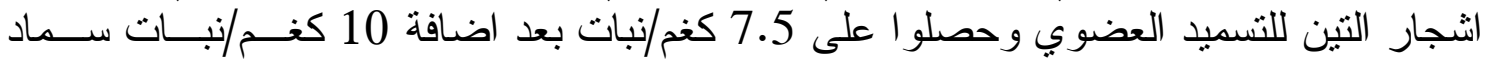

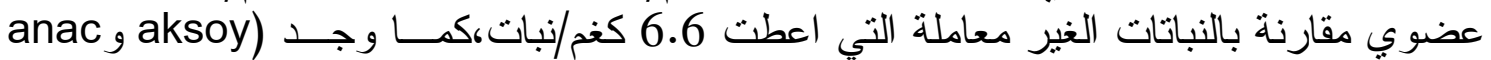
1993) علاقة بين محتوى التربة من العناصر المعدنية و التغذية الجيدة للنبات وبين نمو و إنتــاج 
التين و اتفق معهما (باثـة 1998) الذي اثـار الى انة للحصول على انتاج جيد من الثمار يــضـاف

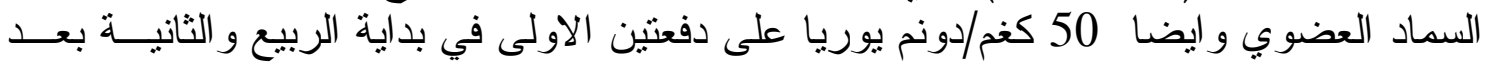

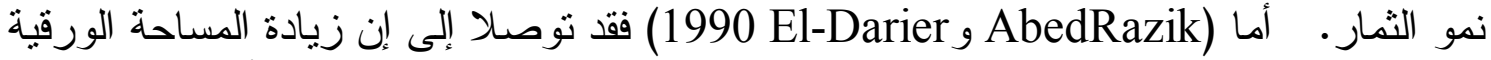

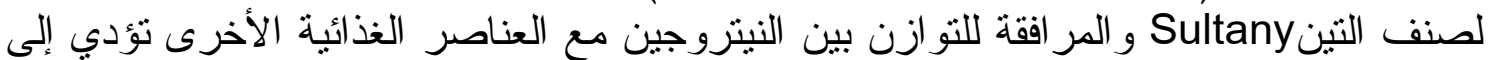

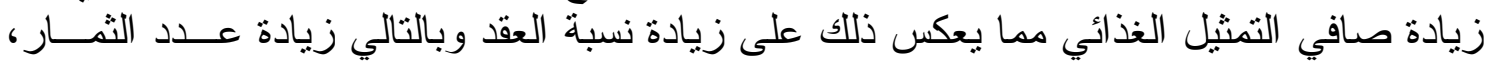
كما حقق (Mehmet و آخرون

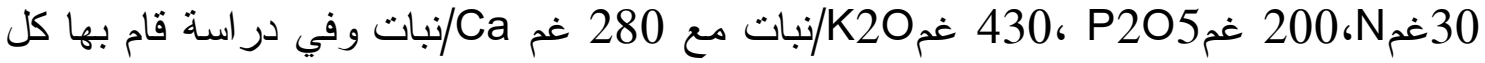
من( Ferrara و 2003 Papa و جدا إن الاختلاف في وزن الثمار يعزى كاســتجابة للتسميد

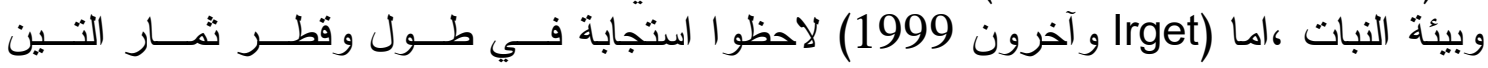

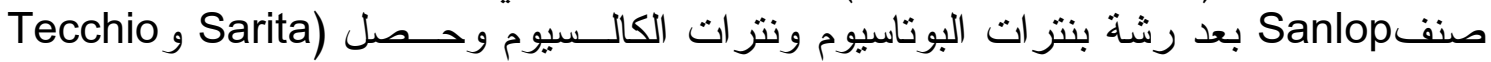

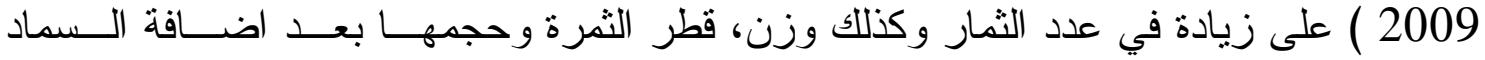

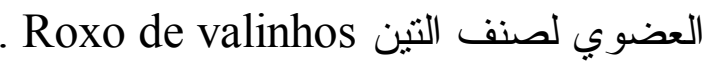

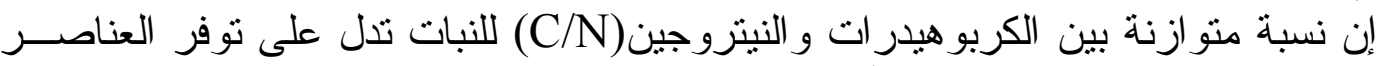

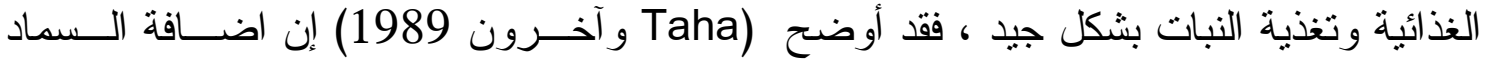
الفوسفاتي و البوتاسي مع اليوريا او كبريتات البوتاسيوم اعطت نسبة C/N جيدة ادت الت الى تحسين

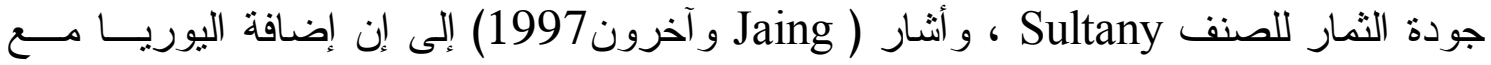

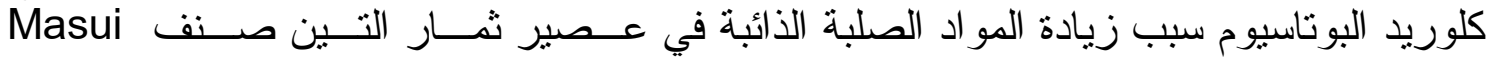

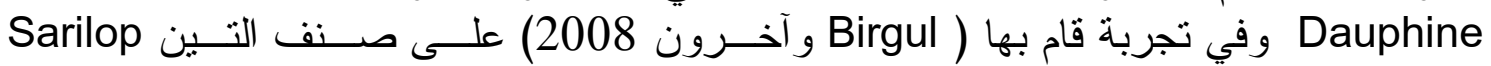

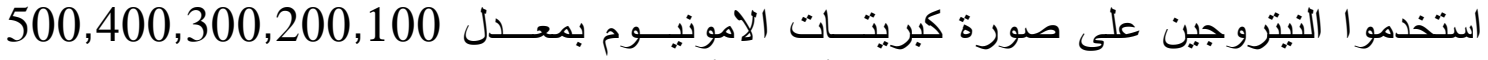
غم/نبات وجدوا إن جرعة 100 غم/نبات أعطت أعلى نسبة للمو اد الصلبة الذائبة لعصير ثــــار

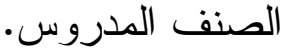

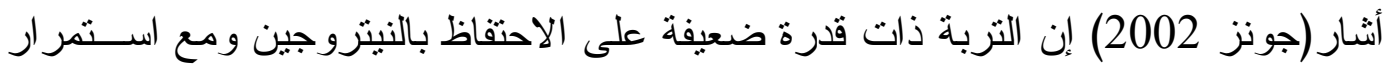

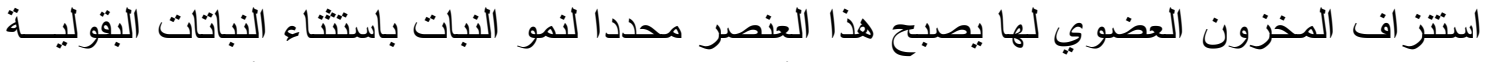

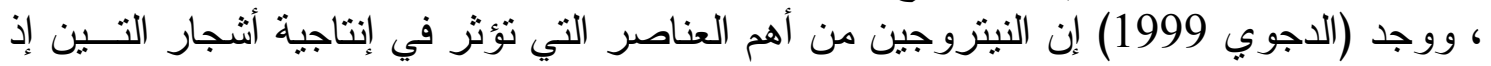

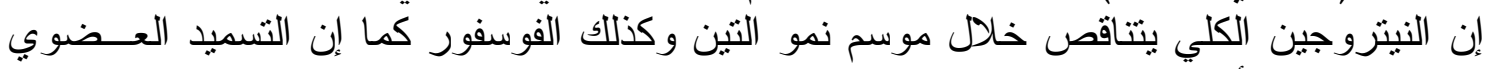

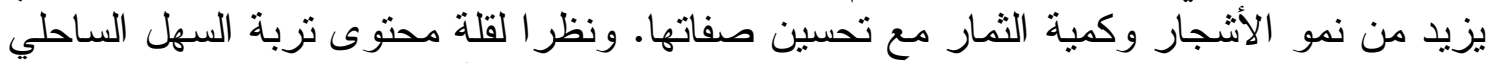

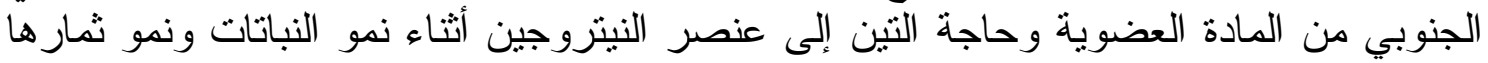

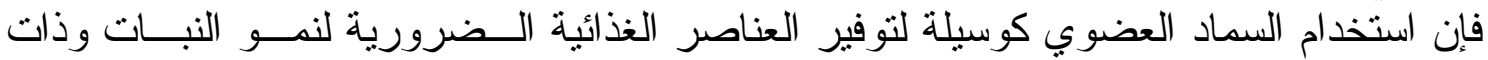

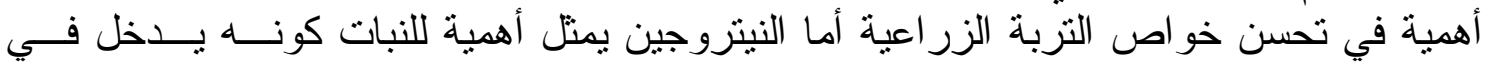

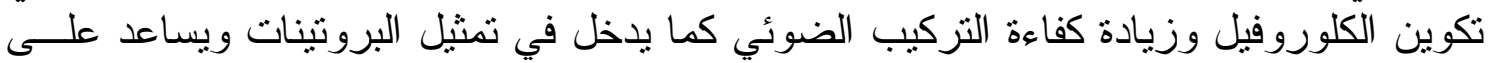

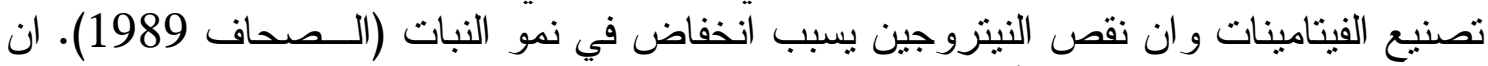

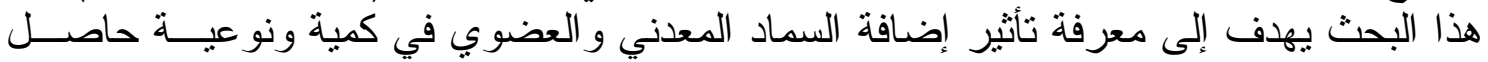
التين صنف أسود سكري. مواد وطر ائق العمل

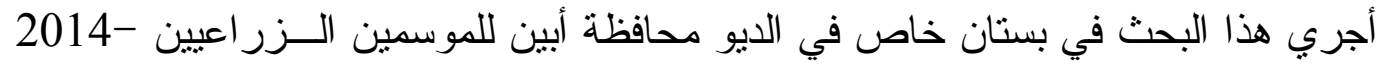

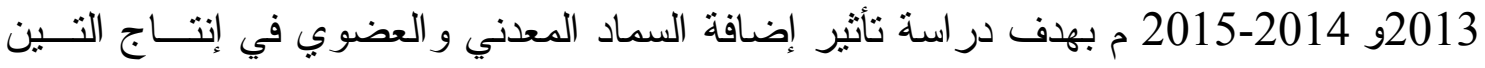

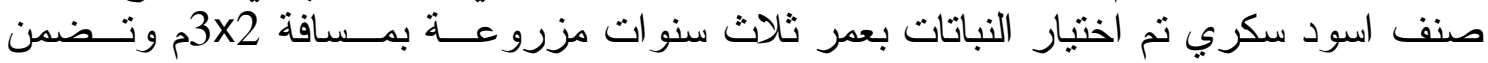
البحث إضافة اليوريا(46 \% نيتزوجين) و السماد الفوسفاتي على هيئة سوبر فوسفات ثناثي (46)

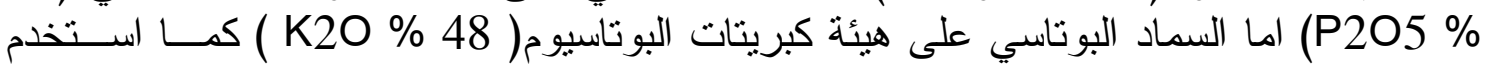
السماد العضوي من مخلفات الاغنام و الابقار للمحصول كيرلات الثاني، وكانت المعاملات كالتالي: 


$$
\begin{aligned}
& \text { 1- المقارنة ( بدون تسميد) • }
\end{aligned}
$$

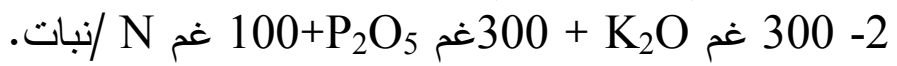

$$
\begin{aligned}
& \text { 3- } 10 \text { كغ سماد ابقار / نبات. } 10 \text { كغ }
\end{aligned}
$$

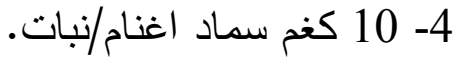

$$
\begin{aligned}
& \text { 5- } 10 \text { كغم سماد ابقار/نبات +100 100 غم N/نبات }
\end{aligned}
$$

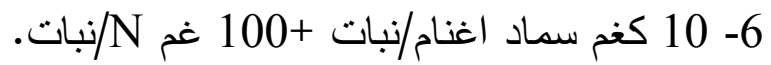

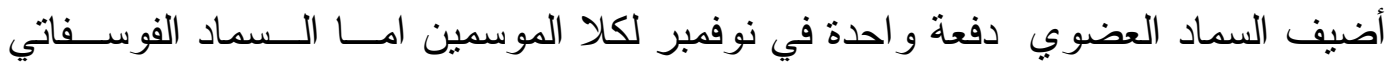

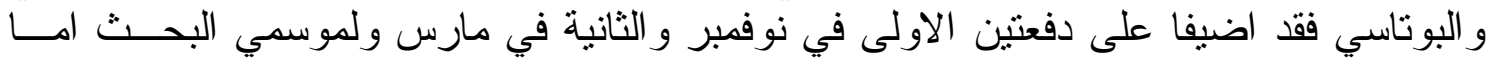

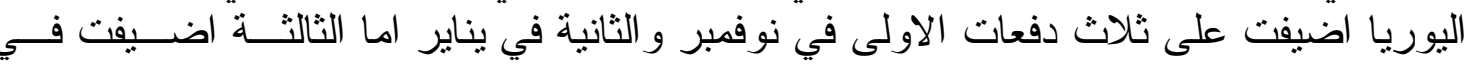

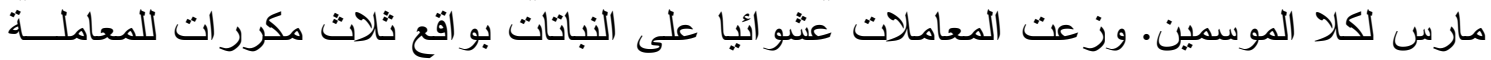

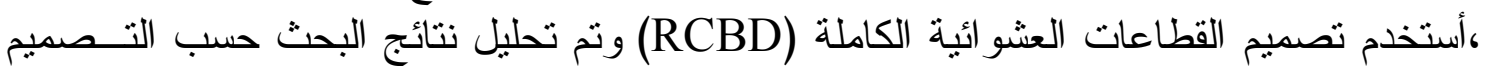

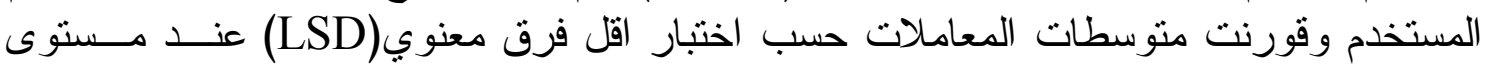

$$
\text { الصفات المدروسة } 5 \text { (بعقوب: 2005). }
$$

1- الحاصل: تم حساب الحاصل بضرب مدرب معدل وزن 15ثمرة X عدد الثمار لكل مكرر. 2- عدد الثمار/نبات: حسبت عدد الثمار عند جني الحاصل.

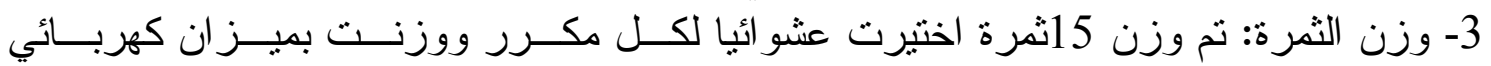

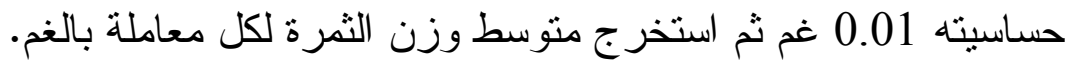

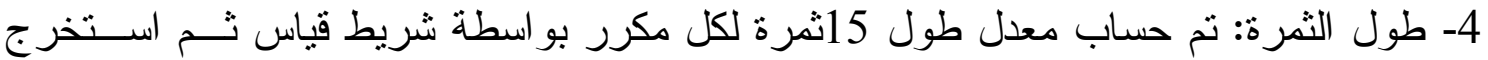
متوسط طول الثمرة لكل معاملة بالسم.

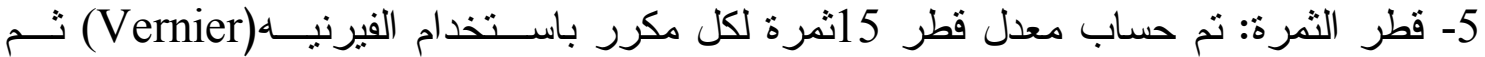
استخر اج متوسط قطر الثمرة لكل معاملة بالسم.

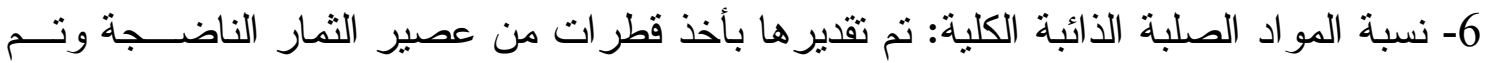
قر اءتها بو اسطة جهاز المكسار ( Hand Refractometer). 7- نسبة الحموضة الكلية: تم حسابها بمعايرة حجم معين من عصير الثمار الناضجة (على أساس

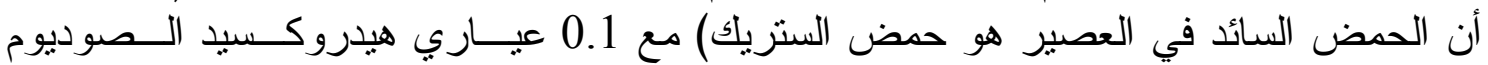
وباستخدام دليل الفينونفتالين (26 Ranganna). حجم القاعدة X عياريتها X الوزن المكافئ لحمض الستريك $100 \mathrm{X}$

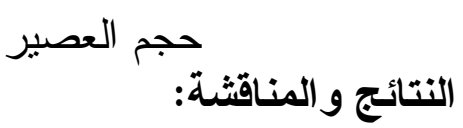

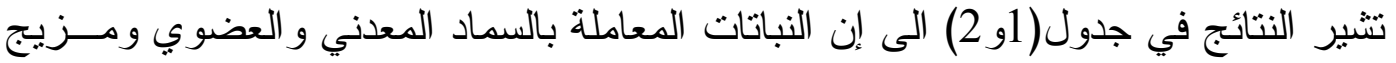

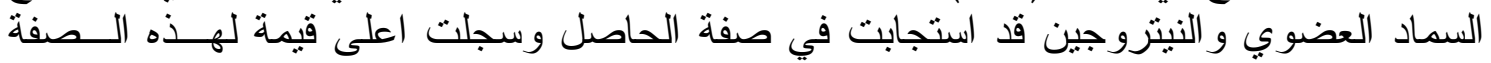

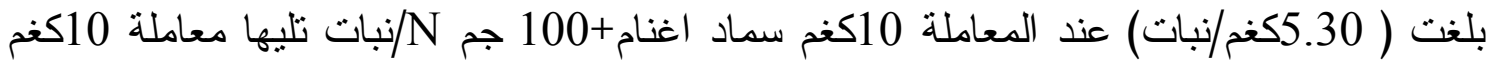

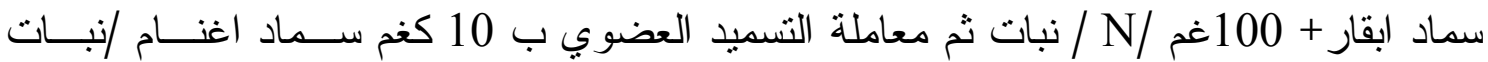

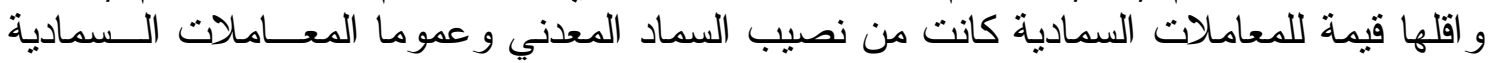

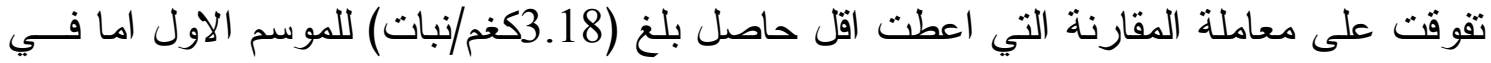

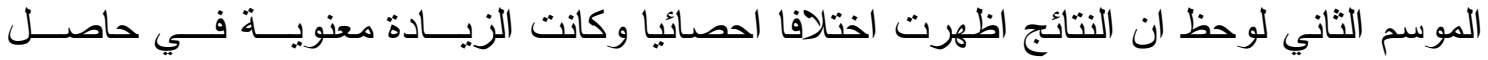
النباتات المعاملة سواء بالسماد المعدني او العضوي و عموما تفوقت المعاملة 10 كغم سماد اغنام 
+ 100غم N/نبات على بقية المعاملات إذ اعطت اعلى حاصل بلغ (54.98 كغم /نبات) مقارنـــة بمعاملة المقارنة التي اعطت اقتل إنل حاصل بلغ (3.54 كغم / نبات).

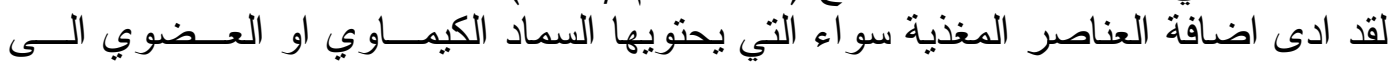

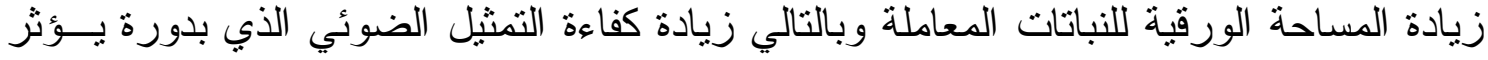

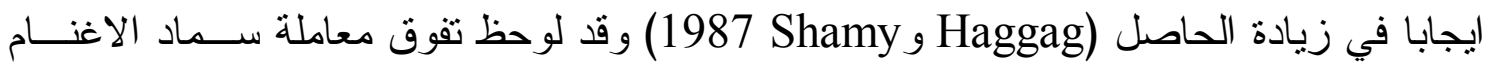

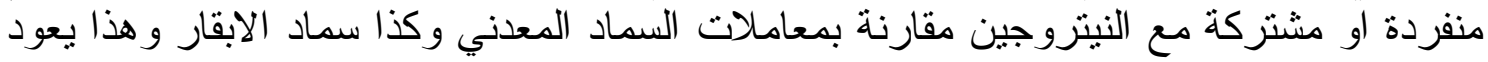

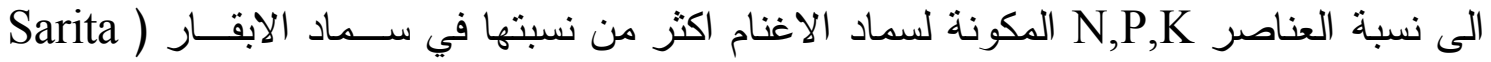

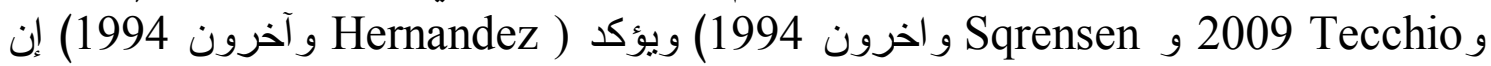

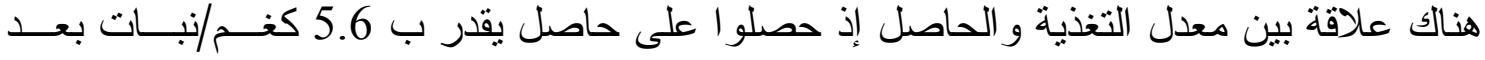

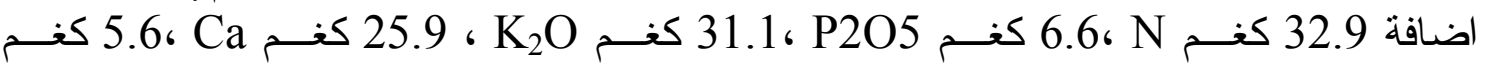
• هكتنار

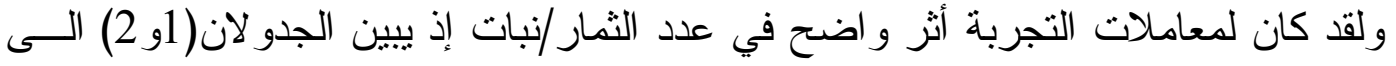

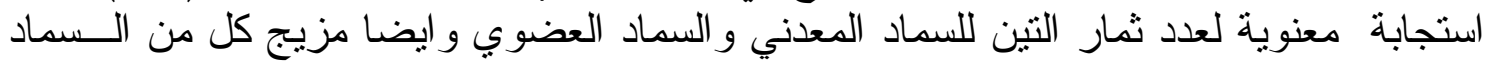

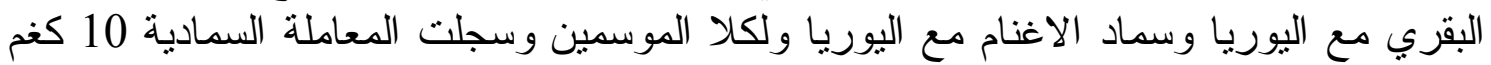

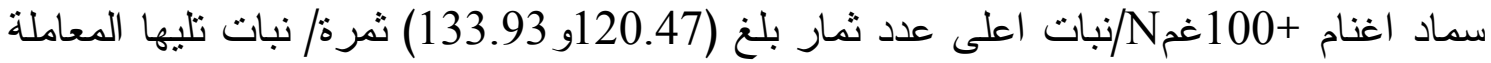

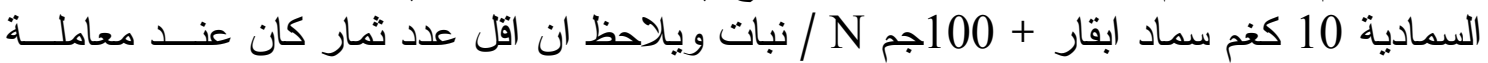

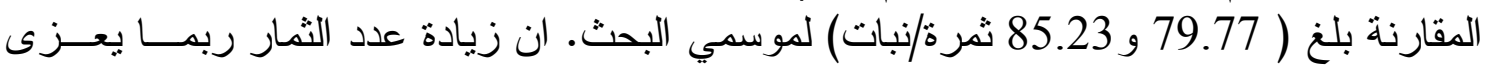

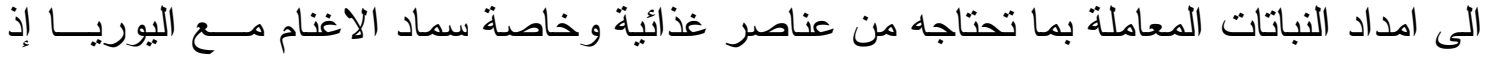

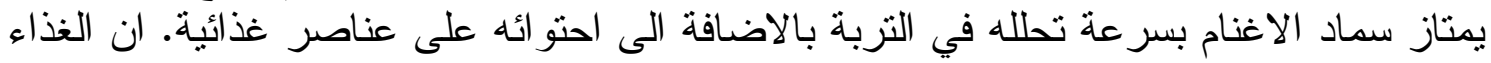

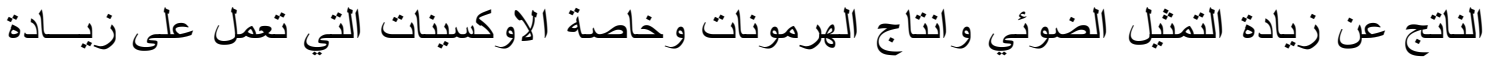
نسبة عقد الثمار وكذلك اشتر الك بعض العناصر مثل النيتروجين و الفوسفور في تكوين مركبــات فئات

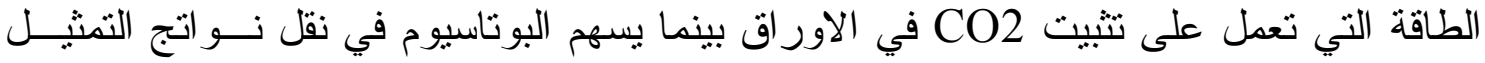
الضوئي الى الثمار (الصحاف 1994) تتفق هذه النتيجة مع (Jinsheng و آخرون 1997) جدول 1. تأثير إضافة NPK و السماد العضوي في بعض صفات التين صنف اسود سكري للموســم

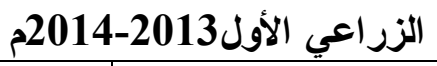

\begin{tabular}{|c|c|c|c|c|c|c|c|}
\hline الحموضة: & 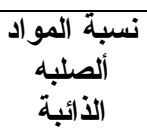 & س قطمة & س الثمرة & غ غزن & تمره / نبات & كفم / نبات & الصفات \\
\hline 0.54 & 11.93 & 4.71 & 4.14 & 39.94 & 79.77 & 3.18 & المقارنة \\
\hline 0.50 & 13.11 & 5.09 & 4.40 & 42.02 & 101.87 & 4.28 & 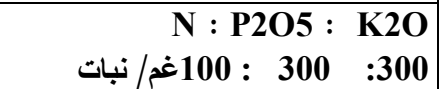 \\
\hline 0.46 & 12.66 & 4.91 & 4.27 & 41.60 & 92.40 & 3.84 & 10 كفم/ بقار \\
\hline 0.40 & 13.91 & 5.24 & 4.56 & 42.74 & 108.33 & 4.63 & 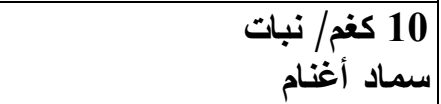 \\
\hline 0.36 & 14.08 & 5.33 & 4.70 & 43.26 & 114.87 & 4.97 & 10 سمفم/ نبات + 100 + 100غم N \\
\hline 0.32 & 14.29 & 5.40 & 4.85 & 44.06 & 120.47 & 5.30 & 10 كماد أغنام + نبات 100 غم N \\
\hline 0.01 & 0.08 & 0.04 & 0.04 & 0.37 & 3.02 & 0.54 & أ. ف. م. عند مستوى 5 \% \\
\hline
\end{tabular}

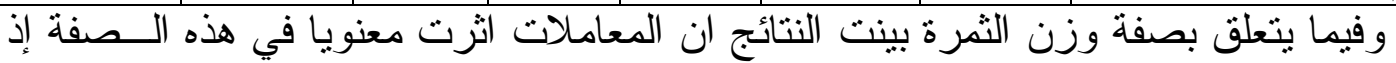

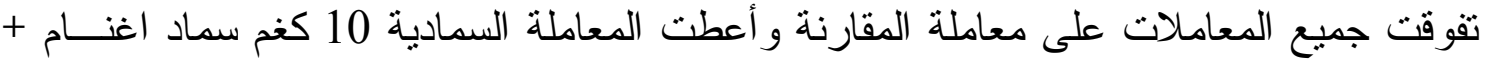




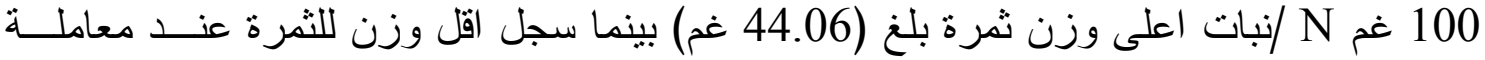

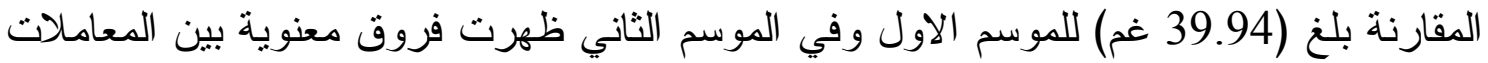

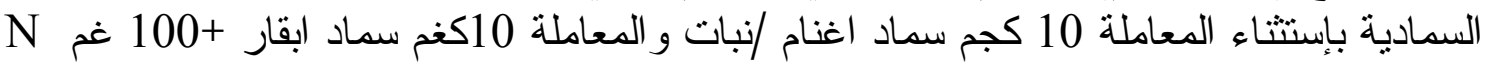

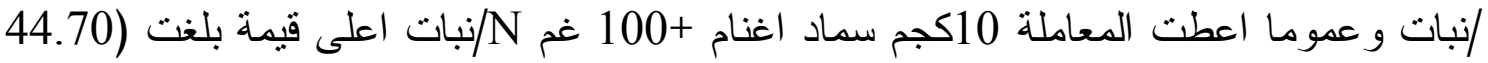

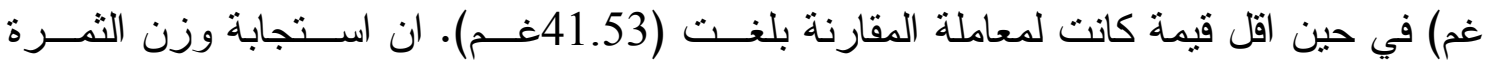

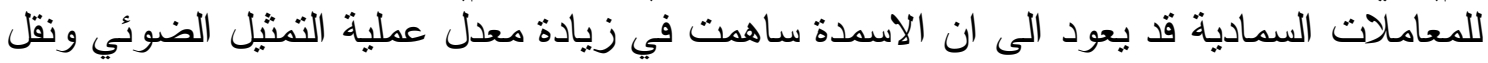

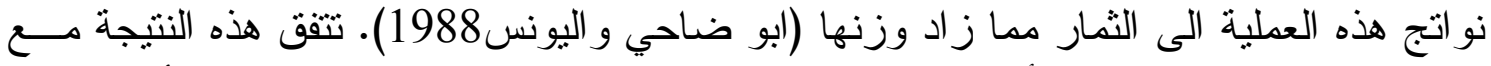
(Eryce)

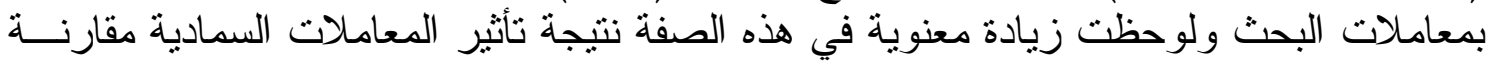

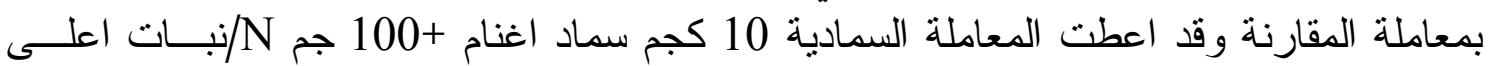

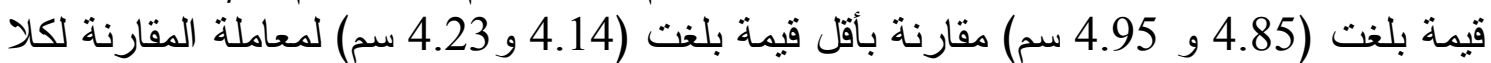

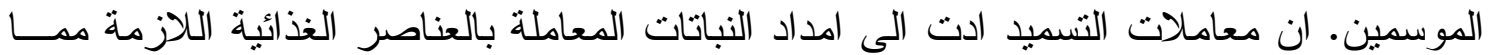

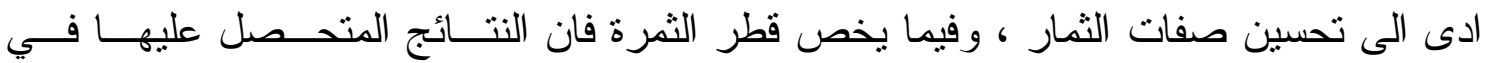

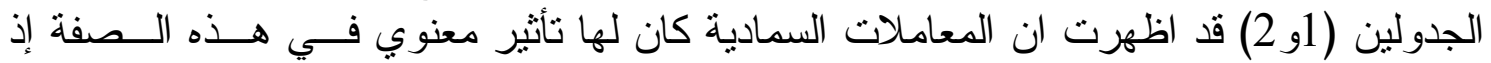

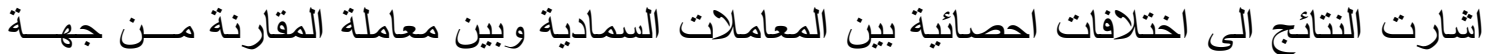

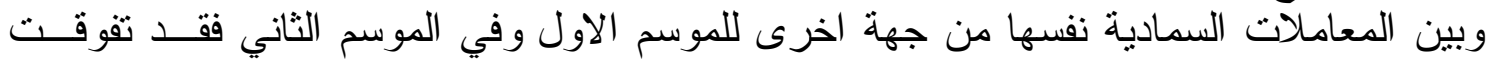

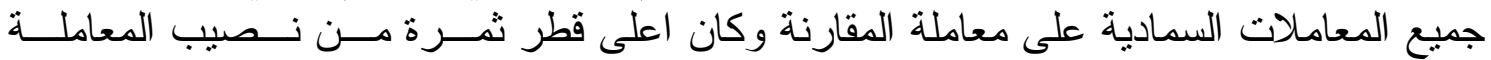

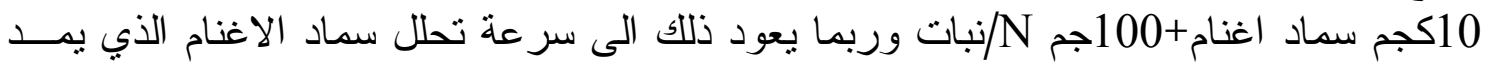

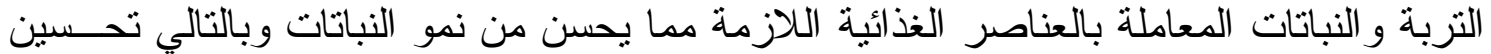
صفات الثمار (2007 kumar).

جدول 2. تأثير إضـافة NPK والسماد العضوي في بعض صفات التين صنف اسود سكري للموسم

\begin{tabular}{|c|c|c|c|c|c|c|c|}
\hline \multicolumn{8}{|c|}{ الزراعي التاسي2014-2015م } \\
\hline الحموضة الكلية & نسبة المبواد الذائة & قالثرة & الثمرة & غ غزن & ثدره / نبات & كفم / نبات & \\
\hline 0.64 & 11.81 & 4.84 & 4.23 & 41.53 & 85.23 & 3.54 & المقارنة \\
\hline 0.47 & 13.40 & 5.17 & 4.52 & 42.70 & 94.03 & 4.01 & $\begin{array}{rr}\text { N : P2O5 : } & \text { K2O } \\
100: 300 \quad 300 & \text { غم/تبات }\end{array}$ \\
\hline 0.51 & 12.78 & 5.01 & 4.41 & 41.97 & 103.07 & 4.32 & $\begin{array}{l}10 \\
\text { كفم/ ابقار نبات }\end{array}$ \\
\hline 0.38 & 13.92 & 5.32 & 4.67 & 43.90 & 117.83 & 5.17 & $\begin{array}{r}10 \\
\text { كفم/ أغنام }\end{array}$ \\
\hline 0.34 & 14.15 & 5.44 & 4.84 & 44.03 & 126.50 & 5.57 & 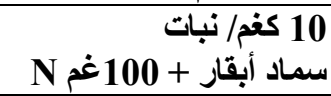 \\
\hline 0.31 & 14.27 & 5.60 & 4.95 & 44.70 & 133.93 & 5.98 & 10 سماد أغنام/ + 100 غمرN \\
\hline 0.03 & 0.09 & 0.05 & 0.02 & 0.58 & 6.80 & 0.02 & أ. ف. م.عند مستوى 5\% \\
\hline
\end{tabular}

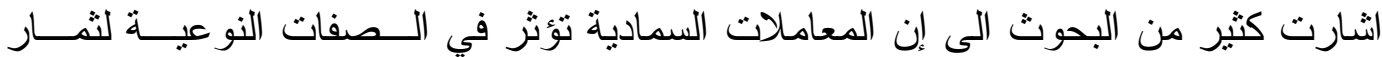

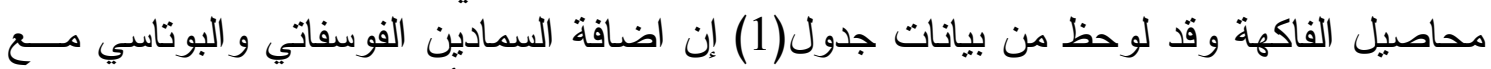

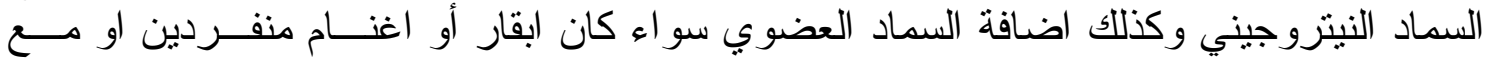

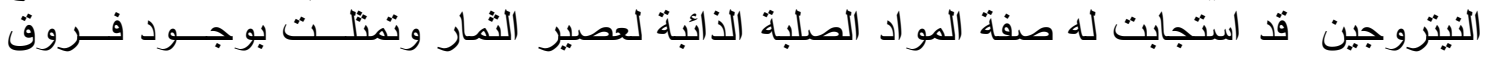
معنوية بين المعاملات وكان هنالك ميل للزيادة في هذه الصفة وصو لا لأعلى نسبة لهــــا (14.29 


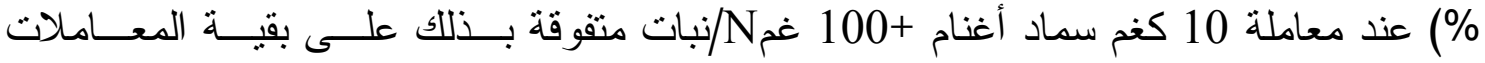
الأخرى في حين اعطت معاملة المقارنة اقل نسبة بلغت (11.93\%) وسلكت ايضا نتائج الموسم

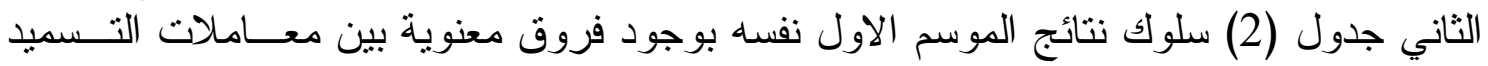

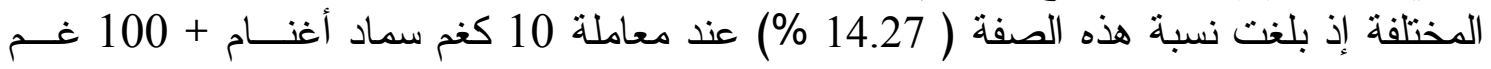
إنبات متقوقة على بقية المعاملات بينما اقل نسبة كانت عند معاملة المقارنة إذ اعطت (11.81

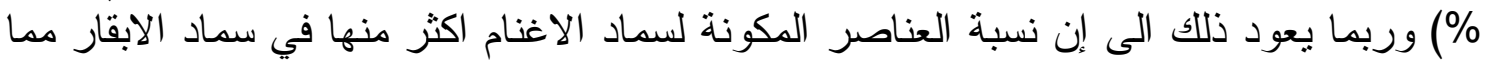
ادى الى امداد النباتات المعاملة باحتياجاتها من العناصر الغذائيــة ( Sarita و 2009 Tecchio

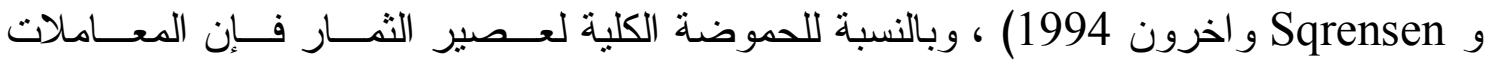

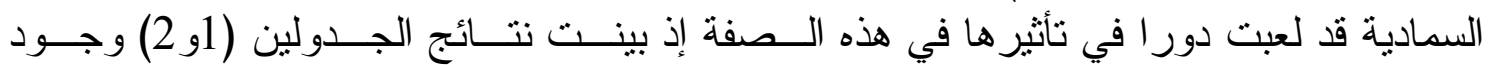
اختلافات احصائية وبفروق معنوية نتيجة التسميد إذ ادت المعاملات السمادية الى تحسين نوعية التهاية

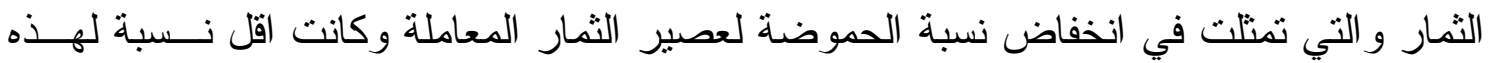

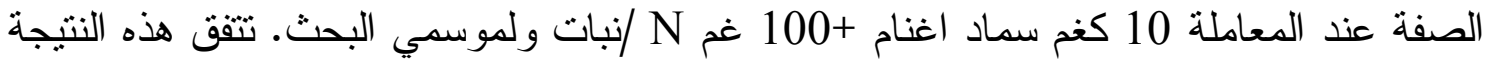

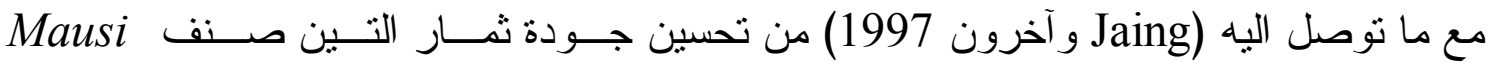

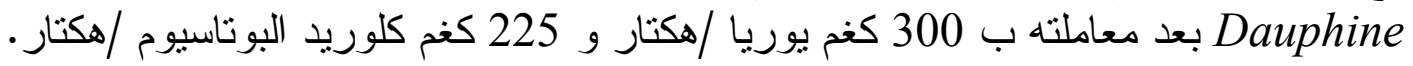

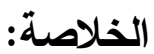

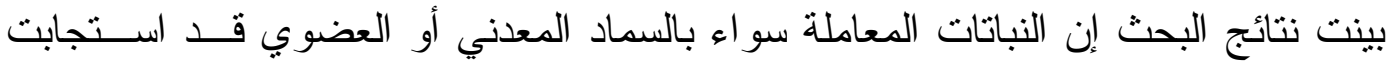

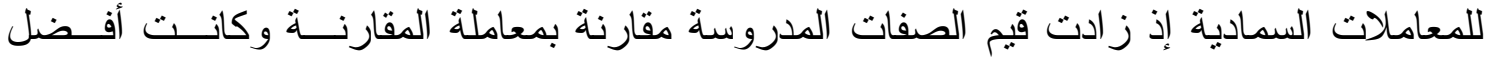

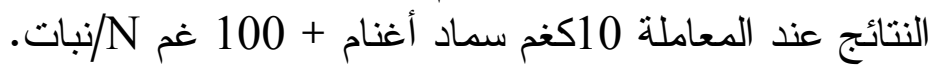

التوصيات:

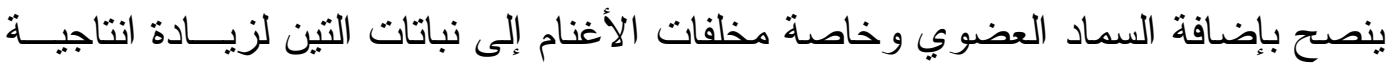

التين وتحسين نو عية الثمار بسبب سرعة تحلله و احتو اءه على العديد من العناصر الغذائية.

المصادر

1- أبو ضاحي ، يوسف محمد ومؤيد احمد اليونس. 1988. دليل تغذية النبات. وزارة التعلــيم العـالي و البحث "العلمي - جامعة بغداد - مطبعة جامعة الموصل التون. العر اق.

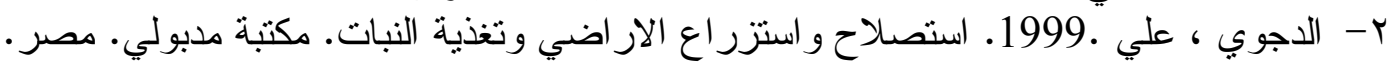

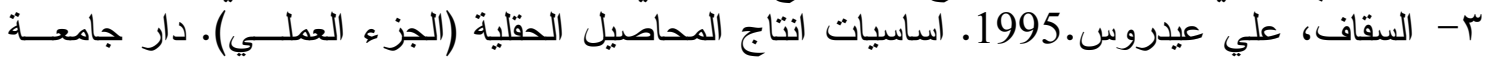

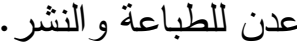

ع - الصحاف، فاضل حسين.1989. انظمة الزر اعة بدون استخدام تربة. وز ارة التعليم العالي و البحــث

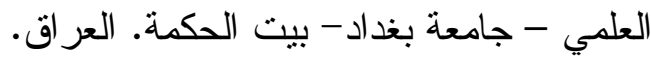

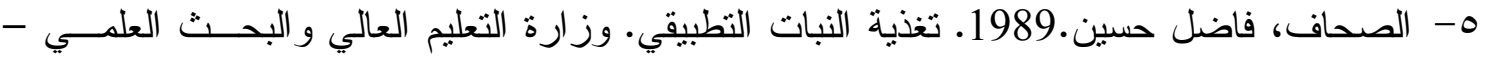
جامعة بغداد- بيت الحكمة. العر اق.

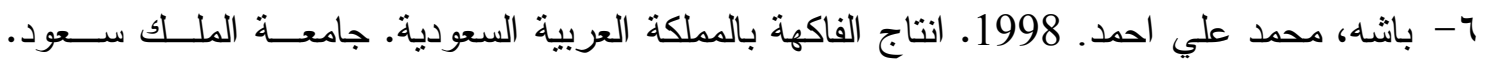
النشر العلمي و المطابع. السعودية.

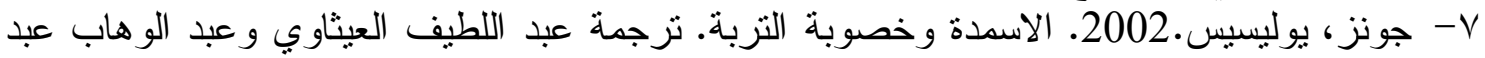
الرزاق. دار الفكر للطباعة و النشر و التوزيع. السعودية.

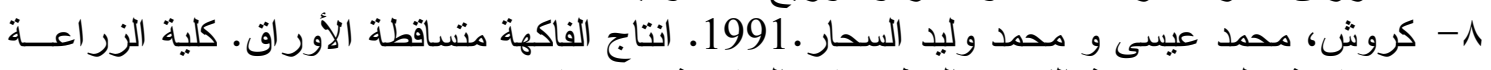
.جامعة حلب. مديرية الكتب و المطبو عات الجامعية. سوريا.

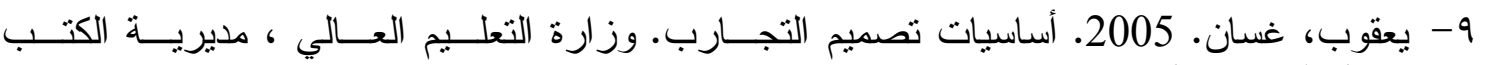
و المطبو عات الجامعية.سوريا.

10-Abdel-Razik, M.S., and S.EL-Darier.1990. Funcutional adaptations of fig trees

(Ficus carica L.) in agroecosy system of the Western Mediterranean desert of Egypt. Qat.Univ.Sci.Jour.11:138-149. 
11-Askoy,U., and D.Anac.1993. Factors affecting quality of fresh and dried fig fruit. Acta Hort. 368:763-766.

12- Askoy,U., H.Z.Can., and S.kara.2003. Fig (Ficus carica L.) selection study for fresh market in Western Turkey.Acta.Hort.605:197-203.

13- Birgul,E., F.Cobanoglu.,B.Sahin.,and A.Belge. 2008. Effect of nitrogen rates on yield and fruit quality of fig. International meeting on soil fertility land management and agroclimatology. P:403-411.

14- Caetano,I.C.S., J.C.Carvalho., and J.M.Jasmim. 2006. Preliminary report on yield productivity and mineral composition of the fig trees as afunction of boron and cattle manure fertilization in Brazil. Fruits. 61:341-349.

15-Eryce, N., H.Caloloclu., S.Audin., B.Cokuysal., D.G.Poulos., C.H.Olympios., and H.Passam. 1995. The effect of $\mathrm{K}$ and $\mathrm{Mg}$ fertilization on some quality characteristics and mineral nutrition of fig. Acta. Hort. 379:199-204.

16- FAOSTAT. 2013. Food and agriculture organization of the United Nation (FAO), FAO STAT, fig production. 2012 and 2013.

17-Ferrara, E., and G. Papa. 2003. Evaluation of fig cultivar for Breba crop. Acta. Hort. 605:91-93.

18-Goziekci, S. 2010. Selection studies on fig in Antalya of Turkey. African Jour. Bio tech. 9(46):7857-7862.

19-Haggag., M.N., and H.A. EL- Shamy. 1987. Response of fig and pome granate fruit trees to NPIC fertilization. Alex J. Agri. Res. 32(3):199-208.

20-Hernandez, F.B.I.,M.A.Suzuki.,J.S.Modesto.,L.S.Correa.,and K. Reichardt. 1994. Response of fig trees orchard under irrigation and nitrogen levels at the Ilha solteira (SP) region. Sci. Agri. 51(1):99-104.

21-Irget,M.E.,S.Aydin., M.Oktay., M.Jutam.,U.Aksoy., and M. Nalbant .1999. Effect of foliar potassium nitrate and calcium nitrate application on nutrient content and fruit quality of fig. plant and soil. Sci., 86:81-86.

22-Jiang, S., Y. Deznu., and Ye.Dz. 1997. Effect of supplementary application of N, K fertilizer on the production and fruit quality of fig trees. J. fruit Sci.14(2):119-120.

23-Jinsheng, Y., Z.MI-Len., J.S. Yang., L.Mi., and Y.H.Zhou.1997. Preliminary report on application rates of single fertilizer with nitrogen, phosphorus and potassium on fig tree. Jiangsu agricultural sciences. 4:47-49.

24-Kumar, A.R. 2007. Studies on integrated nutrient and post harvest 27 Management of fig (Ficus carica L.). Thesis submitted to the university of agricultural sciences, Dharwad in partial fulfillment of the requirement for the degree of $\mathrm{Dr}$. $\mathrm{Ph}$. In Horticulture.

25-Mehmet, E.I.,U.Aksoy., B.Okui., A.r.Ongun., and M.Tepecik. 2008. Effect of calcium based fertilization on dried fig (CV.Sanlop) yield and quality. Sci. Hort.118(4):308-313.

26- Ranganna. S. 1977. Manual of analysis of fruit and vegetable products - Tata - Mc graw - Hill publishing co. New Delhi. pp.603.

27-Sarita, L. and M.A.Tecchio. 2009. Cattle manure fertilization increases fig yield. Sci. Agri (Piracicba Braz.). 66(6):137-149.

28-Sqrensen, P.; E.S. Jensen., and N.E. Nielsen. 1994. The flate $\mathrm{N}^{15}$ - labelled organic nitrogen in sheep manure applied to soils of different texture under field conditions. Plant and soil. 162: $39-47$.

29-Taha, M.H., H. Shahein, and A.M.Attalla.1989. Effect of soil or foliar fertilization trails on vegetative growth, yield and nutritional status of fig trees grown at the North-Western coast of Egypt. Alex. J. Agri. Res. 34(2):67-80. 
عر عبيـ محدد و حسبن علي سالم، 7 ب.

\section{Effect of N, P and K \& Manure Fertilizer on some Fruit Characteristics Fruits of Fig (Ficus carica L.) cv. Esswed Succary Omer Obeid Mohammed ${ }^{1}$ and Hussein Ali Salim ${ }^{2}$}

${ }^{1}$ Department of Life Sciences - College of Education, University of Aden Zanzibar ${ }^{2}$ Ministry of Agriculture and Irrigation, Agriculture Department, the province of Abyan

\section{Abstract}

This study aims to evaluate the effects of N, P and $\mathrm{K}$ and manure fertilizer on fig trees cv. Esswed Succary. Experiment was carried out on private orchard at El-Dew, Abyan Governorate growing seasons 2013-2014 \& 2014-2015. A randomized complete block design was installed with $100 \mathrm{~g} \mathrm{~N}, 300 \mathrm{~g} \mathrm{P}_{2} \mathrm{O}_{5}, 300 \mathrm{~g}$ $\mathrm{K}_{2} \mathrm{O} /$ plant, $10 \mathrm{~kg} /$ plant cow manure, $10 \mathrm{~kg} /$ plant sheep manure, $10 \mathrm{~kg}$ cow manure $+100 \mathrm{gm} \mathrm{N} /$ plant, $10 \mathrm{~kg}$ sheep manure $+100 \mathrm{~g} \mathrm{~N} /$ plant with three replicates. According to the statically analysis there were significant effects of fertilization treatments led to increase fruit yield, fruit number per tree, fruit weight, length and diameter of fruit. The best results were obtained from $10 \mathrm{~kg}$ sheep manure $+100 \mathrm{~g} \mathrm{~N} /$ plant.

Keywords: Fig, mineral nutrition, organic manure. 